\title{
Influence of capping layer and atomic interdiffusion on the strain distribution in single and double self-assembled InAs/GaAs quantum dots
}

\author{
Mou Yang, S. J. Xu, ${ }^{\text {a) }}$ and Jian Wang \\ Department of Physics, The University of Hong Kong, Pokfulam Road, Hong Kong, \\ People's Republic of China
}

(Received 24 September 2007; accepted 16 January 2008; published online 29 February 2008)

\begin{abstract}
The strain distribution in single and double self-assembled InAs/GaAs quantum dots is theoretically investigated by using a valence-force-field model. The results show strong influence of the capping conditions on the strain distribution in individual and stacked dots with wetting layers. In particular, the intermixing of atoms is incorporated into the strain calculations, leading to a conclusion that the atomic intermixing can notably modify the strain profiles near the interfaces of the stacked dot system. (C) 2008 American Institute of Physics. [DOI: 10.1063/1.2841065]
\end{abstract}

Self-assembled quantum dots (QDs) have been extensively studied in recent years due to their potential applications in a variety of optoelectronic and photonic devices. As these QDs are formed by the strain in Stranski-Krastanow growth processes, the strain plays an essential role in the spontaneous formation of self-assembled QDs and a detailed knowledge of the strain distribution is helpful for the development of high quality and uniform QDs. ${ }^{1-3}$ On the other hand, electronic structures and optical properties of the dots are also affected by the strain and, thus, strain control is regarded as an important means to modify emissive wavelengths of dots to meet the application. ${ }^{4}$ Changing the growth conditions of the capping layers (CLs) is a common method to tailor the strain distribution within and around the QDs ${ }^{4,5}$ and, thus, the optical properties. ${ }^{6-8}$ Moreover, atomic interdiffusion during both the growth of dots and barrier/capping layers and the postgrowth thermal annealing processes have significant influences on the strain, morphologies, and optical properties of self-assembled QDs. ${ }^{9-13}$ Therefore, it is highly desirable to gain a better understanding of the strain in the self-assembled QD systems. While precise experimental determination of the strain in nanometer scale QD systems remains challenging, ${ }^{14}$ at the moment, numerical simulation is a major approach to analyze the strain in QD systems. The valence-force-field (VFF) model ${ }^{15}$ is recognized as a powerful method for the strain calculation since it overcomes the disadvantages of the analytic method ${ }^{16}$ and can be applied to cases under extreme circumstances such as high pressure. ${ }^{17-22}$ However, most of the applications focused on buried QD systems and did not take composition mixing into account. In this letter, we employ the VFF model with the Keating potential ${ }^{15}$ to calculate the strain distribution in selfassembled InAs/GaAs QD systems with different capping conditions and intermixing composition profile. We find that the composition intermixing smears the sharp features of the strain profile within the thickness of the order of the diffusion length near the interface. For stacked dot systems, the individual strain components in the upper QD is found quite sensitive to the thickness of spacing layer (SL), while the lower one is not. The CL above the upper QD affects both

\footnotetext{
${ }^{\text {a) }}$ Author to whom correspondence should be addressed. Electronic mail:
} sjxu@hkucc.hku.hk the upper and lower dots but some strain components in the lower QD remain almost unaffected.

It has been well recognized that there is a strong composition intermixing between QDs and surrounding matrix during the thermal annealing process ${ }^{11,12,23}$ and even the growth of CL. ${ }^{9}$ To take this effect into account, we need to determine the spatial redistribution of the composition after the thermal annealing using the standard diffusion equation, $(\partial / \partial t) n(\mathbf{r}, t)=D \nabla^{2} n(\mathbf{r}, t)$, where $D$ is the diffusion coefficient and $n(\mathbf{r}, t)$ is the composition of InAs at position $\mathbf{r}$ for the diffusion time $t$. The initial condition of the equation is defined as $n(\mathbf{r}, 0)=1$ at all sites inside the QD and wetting layer (WL). By introducing a new variable $l=\sqrt{2 D t}$, the diffusion equation can be transformed to $(1 / l)[\partial n(\mathbf{r}, l) / \partial l]=\nabla^{2} n(\mathbf{r}, l)$. For a given diffusion length $L$, corresponding to the diffusion time $T=L^{2} / 2 D$, the composition distribution can be obtained. After the composition redistribution is determined, the spatial-site dependency of the strain-related material parameters is calculated. Finally, the VFF calculation can be carried out.

According to the Keating's VFF model, ${ }^{15}$ the strain energy can be expressed as a function of atomic positions. For GaAs and InAs which have zinc blende crystalline structure, the strain energy can be written as ${ }^{19}$

$$
\begin{aligned}
U= & \frac{1}{2} \sum_{n, i} \frac{3}{8} \frac{\alpha}{d_{0}^{2}}\left(\mathbf{r}_{n}^{i} \cdot \mathbf{r}_{n}^{i}-d_{0}^{2}\right)^{2} \\
& +\frac{1}{2} \sum_{n, i, j \neq i} \frac{3}{8} \frac{\beta}{d_{0}^{2}}\left(\mathbf{r}_{n}^{i} \cdot \mathbf{r}_{n}^{j}+\frac{1}{3} d_{0}^{2}\right)^{2},
\end{aligned}
$$

where $\mathbf{r}_{n}^{i}(i=1,2,3,4)$ is the $i \mathfrak{t h}$ vector between different atoms in the tetrahedral unit cell labeled by $n, d_{0}$ is the equilibrium interatomic bond length, and $\alpha$ and $\beta$ are the bondstretching and bond-bending force constants, respectively. $\alpha$ and $\beta$ can be related with the quantities shown in Table I as

TABLE I. Material parameters used in the present calculations (Ref. 18).

\begin{tabular}{ccc}
\hline \hline Quantity & Units & Values for $\mathrm{In}_{x} \mathrm{Ga}_{1-x} \mathrm{As}$ \\
\hline$a_{0}$ & $\mathrm{~nm}$ & $0.56503+0.04050 x$ \\
$C_{11}$ & $\mathrm{GPa}$ & $118.8-35.5 x$ \\
$C_{12}$ & $\mathrm{GPa}$ & $53.8-8.5 x$ \\
\hline \hline
\end{tabular}



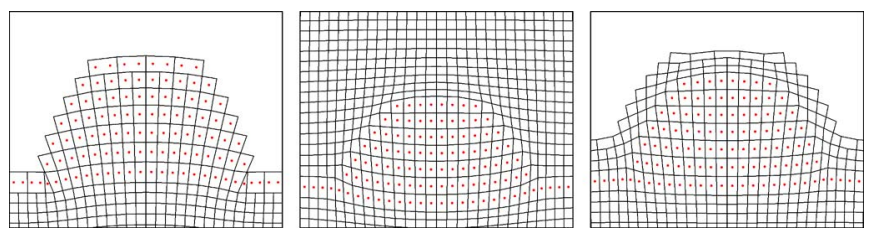

FIG. 1. (Color online) A sketch of the atom positions in the individual InAs QD. Left: a surface QD; middle: buried; right: partially capped.

$\alpha=\left(C_{11}+3 C_{12}\right) d_{0} / \sqrt{3}, \beta=\left(C_{11}-C_{12}\right) d_{0} / \sqrt{3}$, and $d_{0}=a_{0} \sqrt{3} / 4$. The positions of all the atoms are determined by minimizing the total strain energy. After all the atomic positions are obtained, the strain distribution can be calculated. ${ }^{19}$ In our calculations, InAs QDs are assumed to have a truncated pyramid shape with faces along [101], [101], [011], and [0 $\overline{1} 1]$ In addition, one monolayer of uniform InAs WL is taken into account. The base length and height of InAs QDs are set as $b=12 \mathrm{~nm}$ and $h=4 \mathrm{~nm}$, respectively. The $x, y$, and $z$ axes are set to be parallel to the [100], [010], and [001] directions, respectively. We have not observed unphysical oscillating solutions in our calculations. ${ }^{20}$

Before presenting quantitative results, let us try to get a qualitative understanding of the strain. Figure 1 schematically shows the cross sections of the surface, buried and partially capped QDs. The solid points represent the positions of the In atoms in the QD and WL. Note that the strains are appropriately exaggerated for clarity. Because the lattice constant of the InAs WL is larger than that of the GaAs substrate material, the WL thus suffers a laterally compressive strain and then becomes arched upward to release its stress for the uncapped case (surface QD). In addition, it also results in that (1) the GaAs beneath the arch region of the WL is strained (i.e., laterally compressive and vertically tensile) and (2) the strain in the QD layers near the WL shares the same strain characteristics as the WL. Because of the free surface of an uncapped QD, the strain in the QD will relax gradually along the $z$ direction. However, some differences in the strain distribution emerge when the QD is buried inside the GaAs matrix, which include: (1) the WL is no longer arched upward but downward because the QD suffered a large downward force exerted by the GaAs material above it, making the material under it compressed, (2) the strain in the QD cannot freely relax, and (3) the capping material near the QD top is vertically compressed and laterally stretched. Clearly, for the partially capped QD, the strain is somewhat like the intermediate situation between the two cases discussed above.

Figure 2(a) shows the strain profiles along the $z$ axis of an InAs QD capped by GaAs CL with different thicknesses. In the QD region, the vertically tensile strain, which originates from the lateral compressive strain in the WL, becomes smaller with increasing CL thickness, while the lateral strain becomes more apparent due to the larger force exerted by the thicker capping material on the side facets. The hydrostatic strain, i.e., $\operatorname{Tr}[e]$, in the QD is compressive, and its absolute value increases as the $\mathrm{CL}$ thickness increases. It is interesting to note that the strain in the CL is very sensitive to its thickness. The lateral and hydrostatic strains in the CL rapidly decrease with the increase of the CL thickness. Figure 2(b) shows the strain profiles along the $z$ axis of an InAs QD system capped by the $\mathrm{In}_{x} \mathrm{Ga}_{1-x}$ As capping materials with different compositions. Because the lattice mismatch between InAs and $\operatorname{In}_{x} \mathrm{Ga}_{1-x} \mathrm{As}$ is not as large as that between InAs and
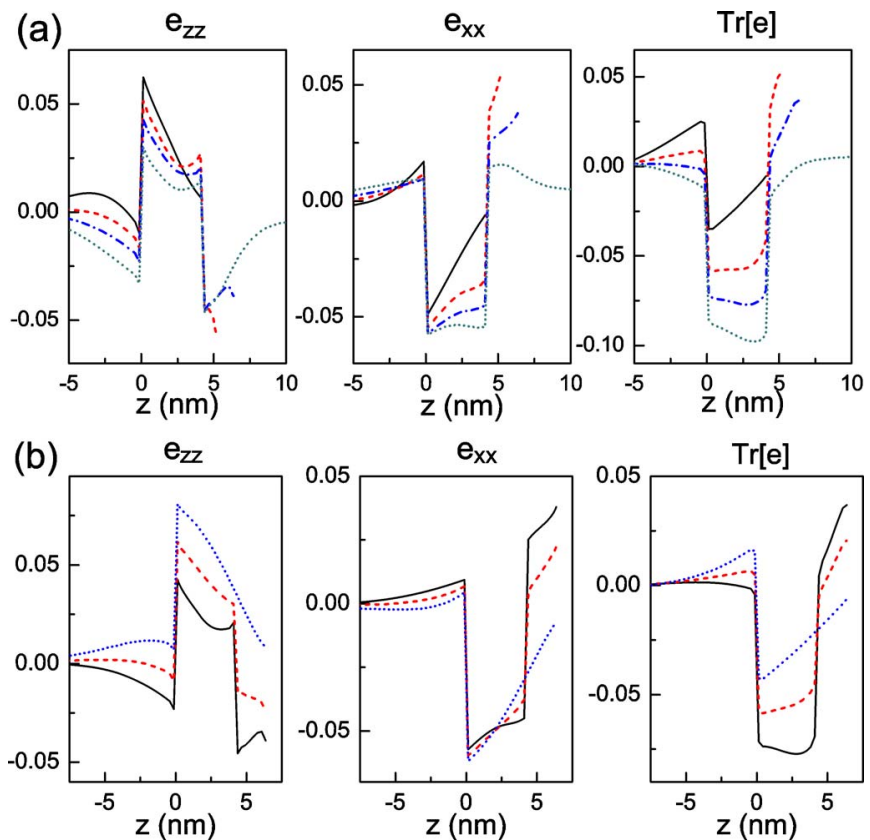

FIG. 2. (Color online) (a) Strain profiles along the $z$ axis in the QD system for different CL thicknesses. The solid, dashed, dash-dotted, and dotted curves correspond to the cases with the CL thicknesses of $\mathrm{cl}=0,2 a_{1}, 4 a_{1}$, and infinity, respectively. Here, $a_{1}=0.565 \mathrm{~nm}$ is the lattice constant of GaAs. (b) Strain profiles along the $z$ axis in the QD system with the $\operatorname{In}_{x} \mathrm{Ga}_{1-x} \mathrm{As}$ capping materials having a fixed thickness of $\mathrm{cl}=4 a_{1}$. The solid, dashed, and dotted curves correspond to the cases with the uniform InAs compositions of $x=0,0.4$, and 1 , respectively. The base length and height of the QD are $b=12 \mathrm{~nm}$ and $h=4 \mathrm{~nm}$, respectively.

$\mathrm{GaAs}$, the more InAs composition in the CL, the larger vertical strain and the smaller hydrostatic strain in the QD, while change of the lateral strain is relatively very small. When the composition of InAs in the CL approaches to 1, the
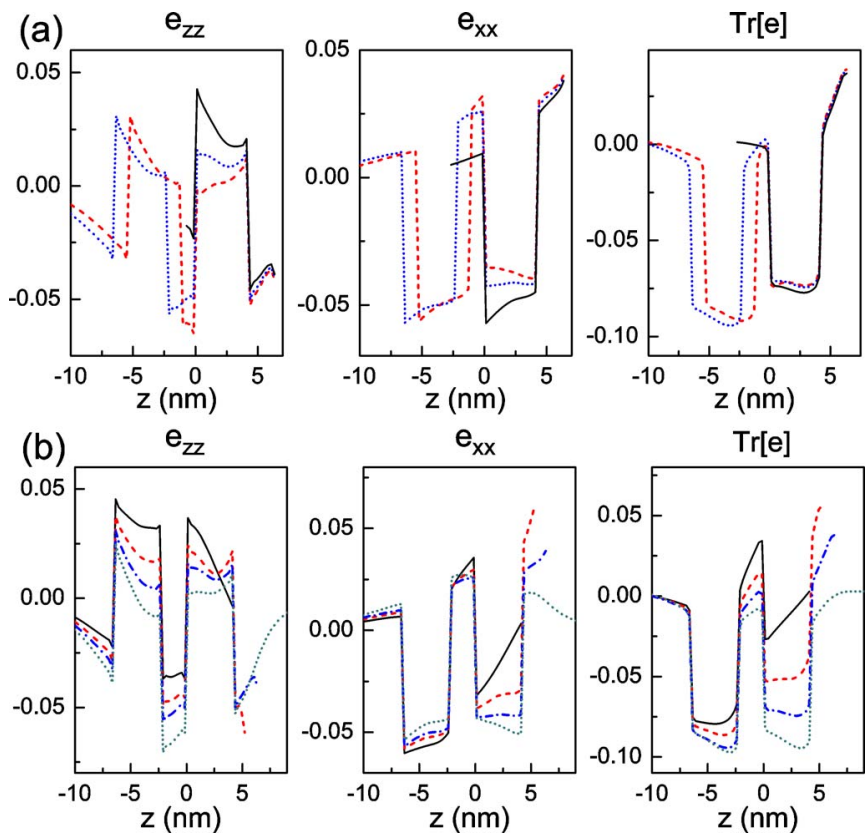

FIG. 3. (Color online) (a) Strain profiles along the $z$ axis in the stacked QD system with the $\mathrm{GaAs}$ CL thickness of $\mathrm{cl}=4 a_{1}$ for different SL thicknesses. The dashed, dotted, and solid curves correspond to the cases with the SL thicknesses of $d=2 a_{1}, 4 a_{1}$, and infinity, respectively. (b) Strain profiles along the $z$ axis in the stacked QD system with the SL thickness of $d=4 a_{1}$ for different CL thicknesses. The solid, dashed, dash-dotted, and dotted curves correspond to the cases with the CL thicknesses of $\mathrm{cl}=0,2 a_{1}, 4 a_{1}$, and infinity, respectively. Other QD parameters are the same as before. 

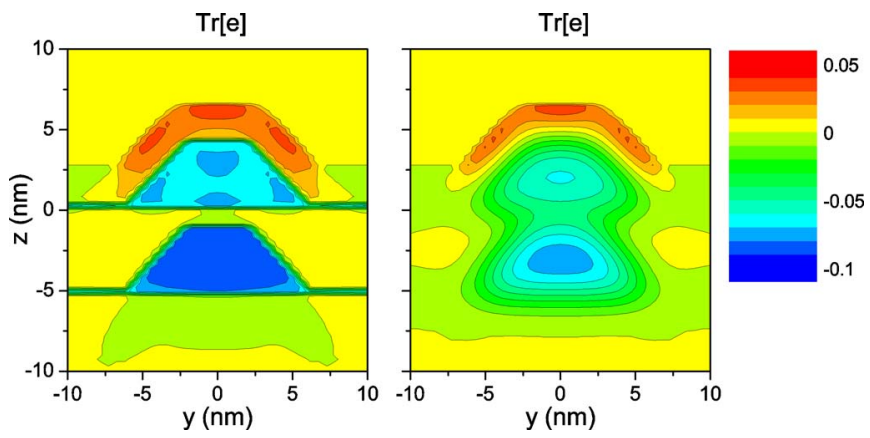

FIG. 4. (Color online) Contour plot of the hydrostatic strains on the $y-z$ plane in the stacked QD system without (left plot) and with (right plot) composition intermixing. The diffusion length $L=2 a_{1}$ is chosen to be equal to the thickness of the SL. Other QD parameters are the same as before.

strain distribution in the QD is reduced to that in a surface QD.

Figure 3(a) shows the strain profiles along the $z$ axis of two stacked InAs QDs separated by the GaAs SL with different thicknesses. The SL thickness is defined as the distance between the top of the lower QD and the bottom of the upper WL. Due to the coupling between the strain fields of the two QDs, ${ }^{24,25}$ the strain profiles in the upper QD are much sensitive to the variation of the SL thickness than the lower one. The lower QD exerts an upward force on the SL, resulting in deformation of the SL and suppression of the strains in the upper QD region. In contrast with the upper $\mathrm{QD}$, the strain profiles in the lower QD are much insensitive to the change of the SL thickness. Figure 3(b) shows the strain profiles along the $z$ axis of stacked double dot systems with different GaAs CL thicknesses. The thicker CL means more downward force exerted by the capping material so that the tensile vertical strain in both the upper and lower QDs decreases and the compressive vertical strain in the SL increases. The variation of hydrostatic strain in the upper QD is like the case of the single QD discussed earlier. However, the lateral strain in the lower QD does not change much. When the atomic interdiffusion is taken into account, the strain profiles of the coupled QD system will be strongly influenced if the diffusion length is comparable with the thickness of the SL. Figure 4 shows a comparison of the hydrostatic strain on the $y-z$ plane in the stacked QD system without and with interdiffusion. The diffusion length is set equal to the SL thickness. It is seen that the atomic interdiffusion greatly modifies the strain profile of the stacked QD system. In particular, the interdiffusion almost irons out the sharp features in the strain distribution at the interfaces. Indeed, the thermally induced atomic interdiffusion is shown to be an efficient way to alter the strain distribution and even electronic structures of self-assembled QDs.

In summary, the strain distribution in InAs/GaAs selfassembled QD systems have been investigated using Keating's VFF model. The results show that the capping conditions as well as the atomic interdiffusion have great impact on the strain profiles of individual and stacked QD system, which are valuable for the controlled growth and device design of self-assembled QDs.

The work was supported by the HK RGC-CERG Grant under Grant No. HKU 7049/04P. The authors would like to thank Dr. Weidong Sheng for his critical reading of this letter.

${ }^{1}$ R. Kunert and E. Schöll, Appl. Phys. Lett. 89, 153103 (2006).

${ }^{2}$ V. Holy, G. Springholz, M. Pinczolits, and G. Bauer, Phys. Rev. Lett. 83, 356 (1999).

${ }^{3}$ B. Yang, F. Liu, and M. G. Lagally, Phys. Rev. Lett. 93, 025502 (2004).

${ }^{4}$ L. Seravalli, P. Frigeri, M. Minelli, P. Allegri, V. Avanzini, and S. Franchi, Appl. Phys. Lett. 87, 063101 (2005).

${ }^{5}$ S. Suraprapapich, S. Panyakeow, and C. W. Tu, Appl. Phys. Lett. 90, 183112 (2007).

${ }^{6}$ W. Sheng and J. P. Leburton, Phys. Rev. B 64, 153302 (2001).

${ }^{7}$ Z. F. Wei, S. J. Xu, R. F. Duan, Q. Li, J. Wang, Y. P. Zeng, and H. C. Liu, J. Appl. Phys. 98, 084305 (2005).

${ }^{8}$ H. B. Wu, S. J. Xu, and J. Wang, Phys. Rev. B 74, 205329 (2006).

${ }^{9}$ S. J. Xu, H. Wang, Q. Li, M. H. Xie, W. J. Fan, and S. L. Feng, Appl. Phys. Lett. 77, 2130 (2000).

${ }^{10}$ Z. M. Wang, K. Holmes, Yu. I. Mazur, and G. J. Salamo, Appl. Phys. Lett. 84, 1931 (2004)

${ }^{11}$ S. J. Xu, X. C. Wang, S. J. Chua, C. H. Wang, W. J. Fan, J. Jiang, and X. G. Xie, Appl. Phys. Lett. 72, 3335 (1998).

${ }^{12}$ H. S. Djie, O. Gunawan, D. N. Wang, B. S. Ooi, and J. C. M. Hwang, Phys. Rev. B 73, 155324 (2006).

${ }^{13}$ H.-Y. Wang, C.-P. Lee, H Niu, C. H. Chen, and S.-C. Wu, J. Appl. Phys. 100, 103502 (2006).

${ }^{14}$ R. Otto, H. Kirmse, I. Häsler, W. Neumann, A. Rosenauer, D. Bimberg, and L. Müller-Kirsch, Appl. Phys. Lett. 85, 4908 (2004).

${ }^{15}$ P. Keating, Phys. Rev. 145, 637 (1966); R. Martin, Phys. Rev. B 1, 4005 (1970).

${ }^{16}$ J. D. Eshelby, Proc. R. Soc. London, Ser. A 241, 376 (1957).

${ }^{17}$ J. W. Luo, S. S. Li, J. B. Xia, and L. W. Wang, Phys. Rev. B 71, 245315 (2005).

${ }^{18}$ O. Stier, M. Grundmann, and D. Bimberg, Phys. Rev. B 59, 5688 (1999).

${ }^{19}$ C. Pryor, J. Kim, L. W. Wang, A. J. Williamson, and A. Zunger, J. Appl. Phys. 83, 2548 (1998).

${ }^{20}$ Y. Kikuchi, H. Sugii, and K. Shintani, J. Appl. Phys. 89, 1191 (2001).

${ }^{21}$ Y. Nabetania, T. Matsumoto, G. Sasikala, and I. Suemune, J. Appl. Phys. 98, 063502 (2005).

${ }^{22}$ H. Shin, J. Kima, Y. Yoo, W. Lee, E. Yoon, and Y. Yu, J. Appl. Phys. 99, 023521 (2006).

${ }^{23}$ I. Kegel, T. H. Metzger, A. Lorke, J. Peisl, J. Stangl, G. Bauer, J. M. García, and P. M. Petroff, Phys. Rev. Lett. 85, 1694 (2000).

${ }^{24}$ H. Heidemeyer, U. Denker, C. Müller, and O. G. Schmidt, Phys. Rev. Lett. 91, 196103 (2003).

${ }^{25}$ N. Nuntawong, J. Tatebayashi, P. S. Wong, and D. L. Huffaker, Appl. Phys. Lett. 90, 163121 (2007). 